\title{
\begin{tabular}{l|l} 
Mitraries & DSpace@MIT
\end{tabular}
}

\author{
MIT Open Access Articles
}

\section{Templated self-assembly of Si-containing block copolymers for nanoscale device fabrication}

The MIT Faculty has made this article openly available. Please share how this access benefits you. Your story matters.

Citation: Ross, C. A. et al. “Templated self-assembly of Si-containing block copolymers for nanoscale device fabrication." Alternative Lithographic Technologies II. Ed. Daniel J. C. Herr. San Jose, California, USA: SPIE, 2010. 76370H-7. (C2010 SPIE.

As Published: http://dx.doi.org/10.1117/12.848502

Publisher: SPIE

Persistent URL: http://hdl.handle.net/1721.1/58560

Version: Final published version: final published article, as it appeared in a journal, conference proceedings, or other formally published context

Terms of Use: Article is made available in accordance with the publisher's policy and may be subject to US copyright law. Please refer to the publisher's site for terms of use. 


\title{
Templated self-assembly of Si-containing block copolymers for nanoscale device fabrication
}

\author{
C.A. Ross ${ }^{\dagger}$, Y.S. Jung, V.P. Chuang, J.G. Son, K.W. Gotrik, R.A. Mickiewicz, J.K.W. Yang*, \\ J.B. Chang, K.K. Berggren*, J. Gwyther**, I. Manners** \\ Massachusetts Institute of Technology, Dept. Materials Science and Engineering; ${ }^{*}$ Dept. \\ Electrical Engineering and Computer Science, Cambridge MA 02139 \\ **University of Bristol, Department of Chemistry, Bristol, BS8 1TS, UK
}

\begin{abstract}
Block copolymers have been proposed for self-assembled nanolithography because they can spontaneously form well-ordered nanoscale periodic patterns of lines or dots in a rapid, low-cost process. By templating the selfassembly, patterns of increasing complexity can be generated, for example arrays of lines with bends or junctions. This offers the possibility of using a sparse template, written by electron-beam lithography or other means, to organize a dense array of nanoscale features. Pattern transfer is simplified if one block is etch resistant and one easily removable, and in this work we use a diblock copolymer or a triblock terpolymer with one $\mathrm{Si}$ containing block such as polydimethylsiloxane or polyferrocenylsilane, and one or two organic blocks such as polystyrene or polyisoprene. Removal of the organic block(s) with an oxygen plasma leaves a pattern of Sicontaining material which can be used as an etch mask for subsequent pattern transfer to make metallization lines or magnetic nanostructures with feature sizes below $10 \mathrm{~nm}$ and periodicity below $20 \mathrm{~nm}$.
\end{abstract}

Keywords: Block copolymer, triblock terpolymer, nanolithography, self-assembly, polydimethylsiloxane, polyferrocenylsilane, pattern transfer

\section{INTRODUCTION}

Thin films of microphase separated block copolymers, which can form patterns consisting of dense arrays of lines, dots, rings and other geometries, are attractive materials for self-assembled nanoscale lithography [1-5]. In this process, a thin film of the polymer is spin-coated on a substrate, and annealed to induce microphase separation. The overall period of the pattern is determined by the chain length of the block copolymer, and the morphology of the pattern depends on the volume fraction of the blocks. For example, in a diblock copolymer, which consists of two covalently bonded immiscible blocks $\mathrm{A}$ and $\mathrm{B}$, microphase separation can produce spheres or cylinders of A in B or of B in A, or alternating lamellae of A and B. Triblock terpolymers, with three different blocks $\mathrm{A}, \mathrm{B}$ and $\mathrm{C}$, can produce a much wider variety of morphologies, for example square-packed or core-shell cylinders or spheres $[6,7,8]$.

Microphase separation is accomplished by annealing, typically at a modest temperature up to $200^{\circ} \mathrm{C}$ for minutes or hours (though in some materials it occurs at room temperature), or by exposing the polymer to a solvent vapor which increases the mobility of the chains. The tendency of the block copolymer to microphase separate is described by its temperature-dependent Flory-Huggins interaction parameter $\chi$ which is related to the enthalpy of mixing of the blocks. In a symmetrical (lamellar) diblock copolymer, microphase separation occurs when $\chi \mathrm{N}>$ 10.5 [9], where $\mathrm{N}$ is the degree of polymerization of the block copolymer. In nanolithography, a high $\chi$ is therefore useful because it enables microphase separation to occur in shorter chain length (lower N) block

${ }^{\dagger}$ caross@mit.edu

Alternative Lithographic Technologies II, edited by Daniel J. C. Herr, Proc. of SPIE Vol. 7637 $76370 \mathrm{H} \cdot$ @ 2010 SPIE $\cdot$ CCC code: $0277-786 \mathrm{X} / 10 / \$ 18 \cdot$ doi: $10.1117 / 12.848502$ 
copolymers, allowing smaller periodicity to be achieved in the final pattern. Additionally, a higher $\chi$ leads to a sharper interface between the blocks, resulting in lower line-edge roughness. A further consideration for block copolymer lithography is the ability to selectively remove one block while leaving the other intact. Organic blocks can demonstrate high etch selectivity, for example in polystyrene (PS) - polymethyl methacrylate (PMMA) block copolymers, the PMMA can be degraded using UV or electron irradiation then removed to leave PS features [10]. However, the remaining PS is itself easily degraded, for example by an oxygen plasma, and has a low glass transition temperature. While such patterns can be used in additive pattern transfer processes, for subtractive pattern transfer processes it may be advantageous for the remaining block to be more robust. For this reason, we select block copolymers containing a metallorganic or Si-containing block such as a polyferrocenylsilane or polydimethylsiloxane (PDMS) and an organic block such as PS. In such materials an oxygen plasma etch removes the PS while oxidizing the Si-containing block, and the remaining pattern forms a durable etch mask. In addition, these hybrid block copolymers often have a high $\chi$ parameter, for example PSPDMS has $\chi=0.26$ [11], compared to $\sim 0.05$ for PS-PMMA at room temperature [12].

In this article, we review nanolithography applications of Si-containing block copolymers, including polystyrene-b-polydimethylsiloxane (PS-PDMS) diblock copolymers and PS-b-polyferrocenylsilane-b-poly(2vinylpyridine) (PS-PFS-P2VP) and polyisoprene-b-PS-b-PFS (PI-PS-PFS) triblock terpolymers. We discuss strategies for templating the self-assembly of these block copolymers to make patterns relevant to electronic devices, and show examples of pattern transfer into functional materials.

\section{METHODS}

PS-PDMS diblock copolymers with total molecular weight $8 \mathrm{~kg} / \mathrm{mol}-52 \mathrm{~kg} / \mathrm{mol}$ were purchased from Polymer Source. PS-PFS-P2VP (94 kg/mol) and PI-PS-PFS $(82 \mathrm{~kg} / \mathrm{mol})$ triblock terpolymers were synthesized by sequential living anionic polymerization $[7,8]$. Films of block copolymers were spin-coated from solution onto oxidized silicon substrates with topographical templates made using electron-beam lithography or interference lithography. The topographical templates consist of shallow trenches etched into the oxide, or posts made from hydrogen silsesquioxane (HSQ) resist [13]. Substrates were typically treated with a brush layer of short-chain hydroxyl-terminated homopolymer prior to spin coating. After spin coating, the block copolymer films were annealed in a vacuum oven at temperatures up to $200^{\circ} \mathrm{C}$ for minutes or hours, or exposed to vapors of a solvent at room temperature.

The annealed films were etched in an oxygen plasma to remove the organic blocks, leaving oxidized PDMS or PFS patterns. Patterns were transferred into a range of metals by overcoating the block copolymer patterns with a metal film, then etching back using a combined chemical and physical reactive ion etch to leave a reversecontrast image [14]. Transfer into silica and into other polymer layers was accomplished using reactive ion etching.

\section{RESULTS}

\subsection{Topographical Templating}

For lithography applications it is essential to control the locations of the microdomains in the block copolymer. This can be done by registering them to a template patterned on the substrate. Both chemical [15-18] and topographical $[7,13,19,20]$ templates have been successfully used for this purpose. Ideally the density of features in the template should be as low as possible so that the template fabrication can be accomplished quickly and at low cost. Fig. 1(a) shows an example of a PS-PDMS (52 kg/mol, 16.5vol\% PDMS) spherical morphology block copolymer templated by a pattern of $10 \mathrm{~nm}$ diameter HSQ posts coated with a PDMS-OH brush [13]. Each post acts as a surrogate PDMS sphere, defining the location of the surrounding spheres which form a close-packed pattern of $40 \mathrm{~nm}$ period. The HSQ posts are arranged in a sparse array, occupying in this example one in 16 of the PDMS sphere lattice sites. The combination of electron-beam lithography and block copolymer selfassembly therefore reduces the writing time by a factor of about 16 times, compared to the time required to write 
a similar pattern composed entirely of HSQ posts. After etching, the HSQ posts and the oxidized PDMS spheres have similar dimensions and are expected to behave similarly in terms of pattern transfer.

Templated self-assembly can be analysed in terms of the commensuration between the equilibrium period $\mathrm{L}_{\mathrm{o}}$ of the microphase-separated block copolymer and the periodicity $\mathrm{L}$ of the template $[13,15,20]$. If $\mathrm{L}$ and $\mathrm{L}_{\mathrm{o}}$ are commensurate, the sphere lattice can form between the posts without strain. For example, if $\mathrm{L} / \mathrm{L}_{\mathrm{o}}=4$, an arrangement such as Fig. 1(a) forms. Commensurability can also occur for non integer $L / L_{o}$, e.g. if $L / L_{o}=\sqrt{3}$, a sphere lattice forms with its axes oriented at $30^{\circ}$ to the axes of the post lattice, so that one sphere forms at the midpoint of a triangle defined by three posts. However, if $\mathrm{L} / \mathrm{L}_{\mathrm{o}}$ is incommensurate, the sphere lattice is strained, and this leads to an energy penalty. By considering the energies of different possible sphere lattices on a given post lattice, the sphere arrangement can be predicted [13]. Computation methods can also predict the selfassembled morphology, for example Fig. 1(b) shows the result of a self-consistent field theory calculation in the case of $\mathrm{L} / \mathrm{L}_{\mathrm{o}}=3.9$. Commensurability is therefore a key consideration for templated self-assembly, not only for sphere lattices but for cylindrical and lamellar microdomains, and for chemically as well as topographically templated self-assembly. For lithographic applications, it is clearly desirable to maximize $\mathrm{L} / \mathrm{L}_{\mathrm{o}}$ in order to reduce the writing time for the template, but as $\mathrm{L} / \mathrm{L}_{\mathrm{o}}$ increases, the energy penalty for forming the 'wrong' microdomain lattice decreases, and defects in the self-assembled lattice become more probable, limiting the effectiveness of very sparse templates.
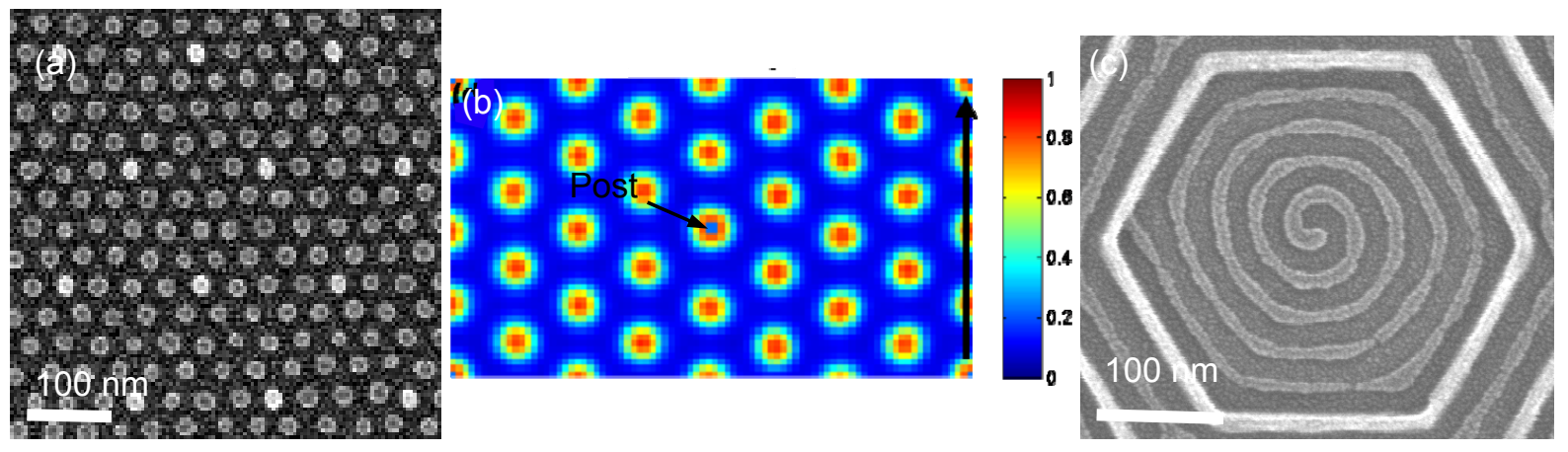

Fig. 1. (a) A lattice of HSQ posts (bright) templates self-assembly of spherical PDMS microdomains with $L / L_{o}=4$. [13] (b) Simulation of self-assembly where $L / L_{o}=3.9$. The red represents the minority domains corresponding to the PDMS spheres in (a) and the blue the majority block, here PS. The posts are blue, at the center (indicated) and the four corners of the computational cell. (c) An example of self-assembly inside a hexagonal pit defined by an electron-beam-written HSQ wall. The PSMS cylinders (light grey) form a spiral pattern; other possible patterns include concentric rings.

The use of a sparse template to create a dense pattern can be extended to other block copolymer geometries and also to non-periodic patterns. For example, in-plane cylinders made from a PS-PDMS $(45 \mathrm{~kg} / \mathrm{mol}, 33 \mathrm{vol} \%$ PDMS) block copolymer can be guided using HSQ posts to form angles or junctions, and even folded into meanders, spirals (Fig. 1(c)) or concentric rings [20] using posts or circular pits. Sparse chemical templates have also been shown effective in templating dense block copolymer patterns [17,21]. Developments in these templating techniques may allow complex dense patterns such as integrated circuit metallization layouts to be deterministically self-assembled from a block copolymer using a template designed with a minimum number of features.

\subsection{Extendability to smaller period}

Smaller period patterns can be achieved using a lower molecular weight block copolymer. Examples of selfassembled patterns with sub- $20 \mathrm{~nm}$ periods and sub-10 nm feature sizes are given in Fig. 2. Linear features of 8 $\mathrm{nm}$ linewidth and $17 \mathrm{~nm}$ period with excellent order were obtained from films of PS-PDMS of molecular weight $16 \mathrm{~kg} / \mathrm{mol}$ and 33\%vol PDMS which were solvent-annealed at room temperature. Unlike the larger molecular weight block copolymers, which order well under toluene vapor annealing, the smaller period structures must be 
annealed in a low vapor pressure of a poorer solvent [22]. It is also significant that the morphology of the microdomains can be changed by different solvents, for example between spheres and cylinders (Fig. 2(a,b)) or cylinders and perforated lamellae [23]. Well-ordered patterns with feature sizes below $10 \mathrm{~nm}$ can be achieved in the PS-PDMS system, suggesting that block copolymer lithography can be extended to the few-nm regime with suitable choice of polymers and processing conditions.
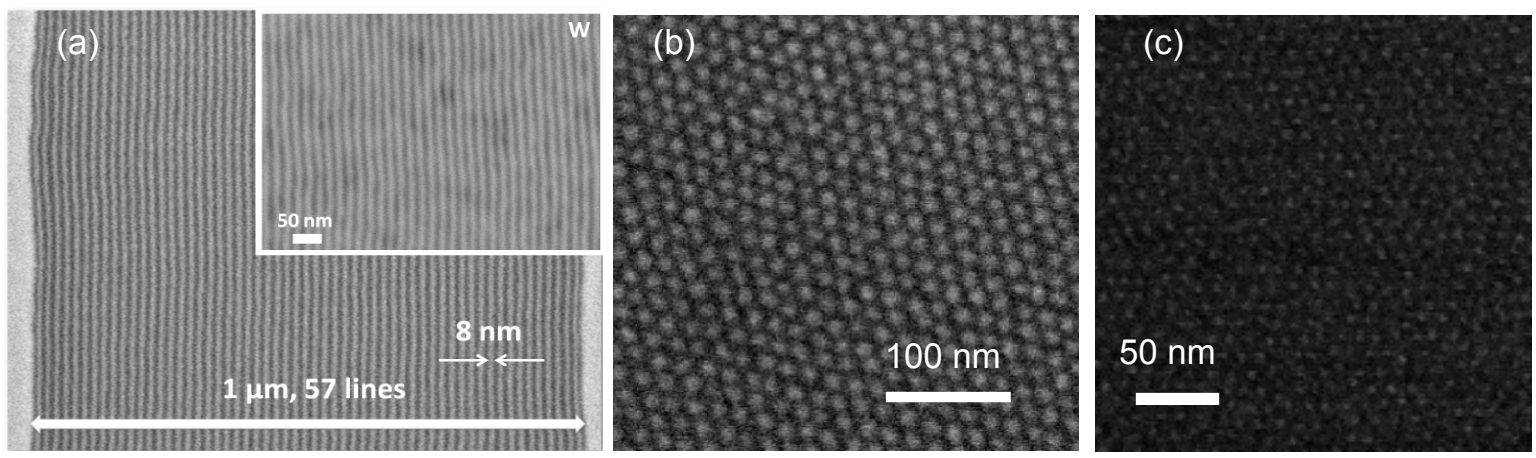

Figure 2. (a) $8 \mathrm{~nm}$ wide, $17 \mathrm{~nm}$ period lines from a $16 \mathrm{~kg} / \mathrm{mol}$ PS-PDMS diblock copolymer, solvent-annealed in acetone. The inset shows the pattern after transfer into tungsten. (b) The same $16 \mathrm{~kg} / \mathrm{mol}$ PS-PDMS self-assembled on a substrate coated with a PDMS brush, solventannealed in dimethylformamide (DMF). The center-to-center distance of the spheres is $17 \mathrm{~nm}$.

(c) Micrograph of self-assembled $8 \mathrm{~kg} / \mathrm{mol}$ PS-PDMS under similar processing conditions to

(b). The center-to-center distance of the spheres is $12 \mathrm{~nm}$.

\subsection{Triblock terpolymers}

Two examples of pattern formation in triblock terpolymers have been investigated, showing square arrays of dots from PI-PS-PFS [7] and close-packed arrays of rings from and PS-PFS-P2VP [8]. Square patterns are of particular interest for structures such as arrays of vias, and cannot easily be achieved from a diblock copolymer which forms close-packed cylinders or spheres. However, in the PI-PS-PFS triblock terpolymer, the minority blocks (PI and PFS) form alternating cylinders with square symmetry. A similar result can also be achieved with a blend of two hydrogen-bonded diblock copolymers, which form a square-packed pattern [24]. Oxygen etching removes the PI and PS, leaving oxidized PFS arrays with period $44 \mathrm{~nm}$. Moreover, these arrays can be templated using topographical steps, or by posts as demonstrated for the PS-PDMS diblock copolymer, as shown in Fig. 3. The direction of the axes of the square lattice with respect to the step edges can be controlled by substrate functionalization (Fig. 3(c,d)).

For the PS-PFS-P2VP, the bulk structure consists of P2VP core- PFS shell cylinders in a PS matrix. Removing the P2VP and PS leaves ring-shaped PFS features. The cylinders can be oriented perpendicular to the top surface of the film by controlling the film thickness and annealing conditions. However, the cylinders typically lie in plane at the substrate-film interface, and the ring pattern therefore cannot be transferred directly by etching an underlying film. Pattern transfer was achieved instead by imprinting using the PFS rings as an imprint stamp [8]. Other examples of core-shell rings have been demonstrated using all-organic triblock terpolymers $[25,26]$. These examples show some of the diversity of geometries that can be achieved using triblock terpolymers. A wide range of geometries remains to be investigated, including lines and spaces of unequal widths, lines with width modulation, or tiling patterns, and self-assembly of these patterns may facilitate the fabrication of new types of devices. 

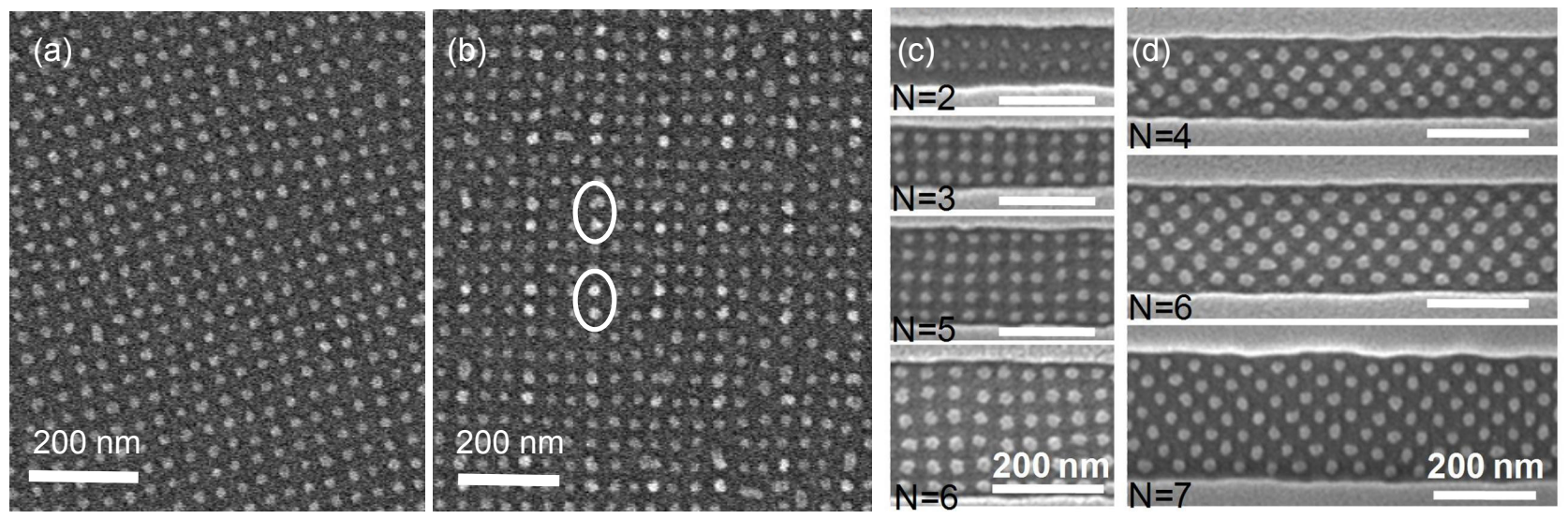

Fig. 2. A square-array of PFS posts from a PI-PS-PFS triblock terpolymer blended with $16 \%$ homopolymer PS. (a) on a smooth oxidized Si substrate with a P2VP brush layer, square patterns with long correlation length can be obtained from a $32 \mathrm{~nm}$ thick film after solvent anneal in chloroform vapor. (b) The locations of the PFS microdomains can be templated using HSQ posts. Here, pairs of posts are fabricated in a square array (some are circled for clarity). The posts are attractive to the PFS block, while the flat substrate is coated with a P2VP brush. (c,d) PI-PS-P2VP / 18\%PS blend film deposited on substrates patterned with shallow trenches, after a chloroform solvent anneal [7]. The orientation of the array depends on the substrate functionalization. In (c) the substrate has no brush layer, while in (d) it has a PS brush.

\subsection{Pattern transfer}

Device applications, including the fabrication of silicon nanowires, transistors, capacitors and Flash memory cells [27,28], patterned magnetic media [29], and conductive polymer sensors [30], have already been described by several groups. Here we describe one example of pattern transfer into metal films using a damascene-like process [14]. Oxidized PDMS line patterns are first prepared from a PS-PDMS diblock copolymer, then overcoated with a layer of metal such as tungsten, cobalt, copper, titanium, etc. The metal film is then etched back using a $\mathrm{CHF}_{3}$ plasma, which physically removes the metal at a low rate. However, once the PDMS patterns are revealed, they are etched quickly. Terminating the etching at this point results in a reverse-contrast metal pattern with a height equal to the height of the original PDMS pattern. The endpoint detection was done using resistivity measurements. This process was found to work well for a range of metals, and arrays of dots, lines and antidots (holes) have been made. For example, Fig. 1(a) inset shows an array of W lines with $9 \mathrm{~nm}$ linewidth and $17 \mathrm{~nm}$ period made in this way.

\section{DISCUSSION AND CONCLUSIONS}

Over the last few years, block copolymer lithography has evolved from a process able to make dense arrays of holes or dots without long range order [31] into a process able to make complex and well-ordered device-like structures of lines or dots which have been transferred into functional materials. The key to this progress has been the use of templating strategies to guide the self-assembly, making not just well-ordered periodic arrays but structures with designed aperiodicities, including the features of interest in device architecture: bends, junctions, and square arrays, for example. In parallel with the development of templating strategies, a more refined approach to annealing and other processing parameters has allowed the orientation, morphology and periodicity of the polymers to be controlled. Much of the published work still involves PS-PMMA diblock copolymers, but Si-containing blocks, organosilicate incorporation, and use of additives that enhance the $\chi$-parameter are extending the range of achievable periodicities. Recent work has shown films with domain patterns with feature sizes of a few $\mathrm{nm}$ and periodicity below $20 \mathrm{~nm}$ [22,32]. By templating such materials, well ordered patterns with sub-10 $\mathrm{nm}$ features are attainable. 
The investigation of triblock terpolymers and other more complex structures is still in its early stages, but offers access to interesting morphologies such as a square array of dots. There is undoubtedly a great deal of progress that can still be made in this area which will enable new morphologies, for example alternating lines of different thickness, or interdigitated structures. In addition, there is considerable opportunity for chemical modification of the blocks to introduce functionality such as electrical conductivity or photosensitivity, or for incorporating nanoparticles or other additives into the blocks. In a recent example [33], a ferromagnetic oxide precursor was introduced into one block and a ferroelectric precursor into another, yielding a multiferroic oxide nanocomposite on annealing.

A number of demonstration devices have been made, such as the nanowire transistor from IBM [27], patterned media from Toshiba [29] and an ethanol vapor sensor [30]. Mass production of devices using block copolymer patterning is still limited, but IBM uses block copolymers in some products to form 'Airgap' dielectric layers. This may change if block copolymer patterning is used to make patterned media. Toshiba, Seagate, Western Digital and Hitachi are seriously investigating block copolymer lithography for next generation hard disk storage devices [34], and this technology may present an entry point for block copolymer patterning. Patterned media requires a single lithography layer (i.e. no registration is necessary) made up primarily of a periodic arrangement of closepacked 'dots', interspersed with servo patterns. Seagate and Hitachi have both proposed a similar manufacturing process in which an e-beam-written large-area template combined with a block copolymer is used to make a master, which is then replicated using nanoimprint lithography. Both e-beam manufacturers and nanoimprint manufacturers are developing suitable tooling for this process.

Block copolymer lithography has developed rapidly in recent years, and seems well enough understood that pilot level manufacturing is feasible. The next challenges include the development of sparse templates to create desired aperiodic features all the way up to self-assembling complete device layouts; extension of templating to sub-10 nm features, and characterization of placement accuracy and size distributions; and strategies to build functionality into the block copolymer instead of just using it as a mask for pattern transfer.

The support of the Semiconductor Research Corporation, the Office of Naval Research and the National Science Foundation for this research is gratefully acknowledged.

\section{REFERENCES}

[1] C.T. Black, R. Ruiz, G. Breyta, J.Y. Cheng, M.E. Colburn, K.W. Guarini, H.-C. Kim, Y. Zhang, IBM J. Res. Dev. 200751605

[2] R. A. Segalman, Mater. Sci. Eng. Rep. 2005, 48, 191

[3] J. Y. Cheng, C. A. Ross, E. L. Thomas, H. I. Smith, Advanced Mater. 2006, 18, 2505

[4] S.B. Darling, Prog. Polymer Sci., 2007, 321152

[5] Joona Bang, Unyong Jeong, Du Yeol Ryu, Thomas P. Russell, Craig J. Hawker, Adv. Mater. 21 4769-92 (2009)

[6] F.S. Bates, Fredrickson, G. H., Phys Today 1999, 52, 32-38

[7] Vivian P. Chuang, C. A. Ross, Jessica Gwyther and Ian Manners, Nano Letts. 9 (12) 4364-4369 (2009)

[8] Vivian P. Chuang, C. A. Ross, Jessica Gwyther and Ian Manners, Adv. Mater. 21(37) 3789-3793 (2009)

[9] F.S. Bates, G.H. Fredrickson, Annual Review of Physical Chemistry 1990, 41, 525

[10] T. Thurn-Albrecht, J. Schotter, C. A. Kastle, N. Emley, T. Shibauchi, L. Krusin-Elbaum, K. Guarini, C. T. Black, M. T. Tuominen, T. P. Russell, Science, 2000, 290, 2126

[11] T. Nose, Polymer 1995, 36, 2243

[12] T.P. Russell, R.P. Hjelm, P.A. Seeger, Macromol. 199023890

[13] Ion Bita, Joel K.W. Yang, Yeon Sik Jung, Caroline A. Ross, Edwin L. Thomas, Karl K. Berggren, Science 321 (5821) p939-943 (2008)

[14] Y.S. Jung and C. A. Ross, Small 5(14) 1654-9 (2009)

[15] L. Rockford, Y. Liu, P. Mansky, T. P. Russell, M. Yoon, S. G. J. Mochrie, Phys. Rev. Lett. 1999, 82, 2602 
[16] S. O. Kim, H. H. Solak, M. P. Stoykovich, N. J. Ferrier, J. J. de Pablo, P. F. Nealey, Nature, 2003, 424, 411

[17] J.Y. Cheng, Rettner, C.T., Sanders, D.P., Kim, H.C. \& Hinsberg, W.D., Advanced Materials 20, 31553158 (2008)

[18] M. P. Stoykovich, M. Muller, S. O. Kim, H. H. Solak, E. W. Edwards, J. J. de Pablo, P. F. Nealey, Science, 2005, 308, 1442

[19] R. A. Segalman, H. Yokoyama, E. J. Kramer, Adv. Mater. 2001, 13, 1152

[20] Y.S. Jung, Jung, W. \& Ross, C.A., Nano Letters 8, 2975-2981 (2008)

[20] J. Y. Cheng, A. M. Mayes, C. A. Ross, Nature Mater. 2004, 3, 823

[21] R. Ruiz et al., Science 321, 936-939 (2008)

[22] Y.S. Jung, J. B. Chang, Eric Verploegen, Karl K. Berggren and C. A. Ross, Nano Letts. (2010)

[23] Y.S. Jung, C.A. Ross, Adv. Mater. 21 2540-45 (2009)

[24] C.B. Tang, Lennon, E.M., Fredrickson, G.H., Kramer, E.J. \& Hawker, C.J., Science 322, 429-432 (2008)

[25] V.P. Chuang, C.A. Ross, P. Bilalis, N. Hadjichristides, ACS Nano 2(10) 2007-2014 (2008)

[26] S. Guo, Rzayev, J.; Bailey, T. S.; Zalusky, A. S.; Olayo-Valles, R.; Hillmyer, M. A., Chem. Mater. 2006, $18,1719-1721$.

[27] C.T. Black, Appl. Phys. Lett. 2005, 87, 163116

[28] C.T. Black, K. W. Guarini, K. R. Milkove, S. M. Baker, T. P. Russell, and M. T. Tuominen, Appl. Phys. Lett. 79, 409 (2001)

[29] K. Naito, H. Hieda, M. Sakurai, Y. Kamata, K. Asakawa, IEEE Trans. Magn. 2002, 38, 1949

[30] Y.S. Jung, Jung, W., Tuller, H.L. \& Ross, C.A., Nano Letters 8, 3776-3780 (2008)

[31] M. Park, Harrison, C., Chaikin, P.M., Register, R.A. \& Adamson, D.H., Science 276, 1401-1404 (1997)

[32] S. Park et al., Science 323, 1030-1033 (2009)

[33] S. Ren, R.M. Briber, M. Wuttig, Appl. Phys. Letts. 93173507 (2008)

[34] X. Yang et al., Presentation DA-02, and D. Kercher, Presentation DA-04, $53^{\text {rd }}$ Conference on Magnetism and Magnetic Materials, Nov. 2008, Austin, TX 